\title{
The Design and Implementation of Multi-function Verification Certificate and Seal in Smart Energy Meter Automatic Test Lines
}

\author{
Liu Shui, Wang Aimin, Zhang Chunqiang, Zheng Zhenzhou \\ Jiangxi Electric Power Research Institute, Nanchang, China \\ liushuilijia@163.net, wimlm-007@163.com,zqc7225@163.net, zhengzz@163.net
}

\begin{abstract}
To design a smart energy meter verification certificate and seal used in automatic testing line, the verification certificate with the seal verification as a whole, after complete all qualified calibration function smart energy meter, automatic verification seal paste in the smart energy meter cover seams on both sides of the table. Verification seal at the upper left of the label is printed with laser technology in the State Grid company logo icon, can be prevented by taking pictures technology imitation this label. Verification seal labels printed right unit verification, verification, verification time and $2 \mathrm{~d}$ barcode seal; Label to paste on the back surface using a two-dimensional grid, glue tear leaves obvious after the words "power theft is illegal" marks, no longer by pasting back to repair. Labels used invisible anti-counterfeiting technology, adopts the two-dimensional bar code encryption algorithm, developed the decryption software, only internal special bar code scanner using a special decryption software can read the correct code value. Other scanner read only is garbled.

Index Terms-Integrated smart energy meter verification seal and certificate; Laser anti-counterfeiting technology; Back glue anticounterfeiting technology; Invisible anti-counterfeiting technology.
\end{abstract}

\section{Introduction}

In order to achieve the concentration verification of measuring instruments, centralized storage, unified distribution and unified supervision, many provinces and municipalities directly under the central government power companies are ongoing construction of provincial measurement center, some measurement center of province and city construction have been completed and start to put into trial operation. All verification qualified smart energy meter must be applied before delivery outbound preventing electricity-stolen seal, paste the verification certificate. On the automatic detection line using traditional preventing electricity-stolen seal requires the use of automatic machine wear sealing line, due to the automatic machine wear sealing line success rate is not high, so the traditional seal is not suitable for use on the automatic verification of assembly line. Because of concentrated gage verification, the amount is very big, if the verification of assembly line using artificial seal and paste certificate, it need to spend a lot of manpower, easy management confusion, the low efficiency, not only affect the automatic detection line speed, and error prone.

\section{The lack of traditional preventing electricity-stolen seal}

Smart energy meter for traditional preventing electricitystolen seal from preventing electricity-stolen and tighten the principle can be divided into wear on the wire fastening type and hangs self-locking type two kinds big. Wear silk fastening type basic principle is: the seal wire through the seal coat first, locking in sealing containers are needed, then fill in the lead and lead seal print clamp will fill material compaction; Hangs self-locking type seal consists of two parts, concave and convex parts, concave and convex parts respectively are matching the out pour it, sealing containers will be inserted into the concave convex, only when the convex pieces on exit, the sealing closed. Traditional preventing electricity-stolen seal has played a large role, but in the smart energy meter automatic calibration line gradually widely used today, there are the following aspects:

1 ) Traditional materials are plastic or metal lead seal, plastic seal materials not environmental protection, if used in the automatic detection line due to the concave and convex parts improper positioning accuracy and pressure size, the success rate is not high; And lead to heavy metals, for sealing body has great harm. And install workload big, wear of seal line need to be very careful, injury body frustrating, if used to automatically test line, automatic wear of seal line success rate is low, therefore, the traditional seal on the smart energy meter automatic detection line is not suitable.

2 ) Traditional preventing electricity-stolen seal structure is simple, anti-counterfeiting technology is poor, easy to imitation and so on shortcomings, can't very well done to prevent the purpose of power theft. Especially when investigate power lead seal authenticity identification, contentious, bring unnecessary trouble for investigate power work.

3 ) Seal asset information management difficulties, in order to facilitate, defensive power management and asset management seal, smart energy meter verification qualified on the smart energy meter automatic detection line of preventing electricity-stolen seal information number need to binding and smart energy meter asset number. Now traditional preventing electricity-stolen seal information number how to automatically read in the management system or a problem. At present only by manual input to the management system, the workload is big, not only is easy to go wrong.

4) Traditional preventing electricity-stolen seal sealing containers and verification certificate of the paste is respectively independently step by step, on the smart energy 
meter automatic detection line requires the implementation of the two independent units to complete each of these two operations. If preventing electricity-stolen seal and verification certificate as an organic whole, can reduce the equipment cost, and can save time, improve efficiency. But traditional seal can display area is lesser; difficult to will be verification, verification, verification certificate required information such as time display. Makes it difficult to seal and certificate integration as a whole.

Therefore, we need to design a suitable smart energy meter verification, automatic calibration lines combined smart energy meter and preventing electricity-stolen seal verification certificate, optimizing anti-counterfeiting technology and seal material, to ensure that seal anti-counterfeiting performance is good, green environmental protection, easy to realize seal asset-like, asset information management.

\section{The Design of Multi-function Verification Certificate}

\section{A Preventing electricity-stolen seal and verification certificate roll into one}

Preventing electricity-stolen smart energy meter automatic verification line verification seal will smart energy meter seal and verification certificate integration for an organic whole, to print and paste the verification seal, verification certificate, as well as the verification seal, correctness verification certificate to print and paste automatic check and so on several ways to synthesize into a process, optimize the process and procedure, and saves equipment investment. Verification seal layout design details below:

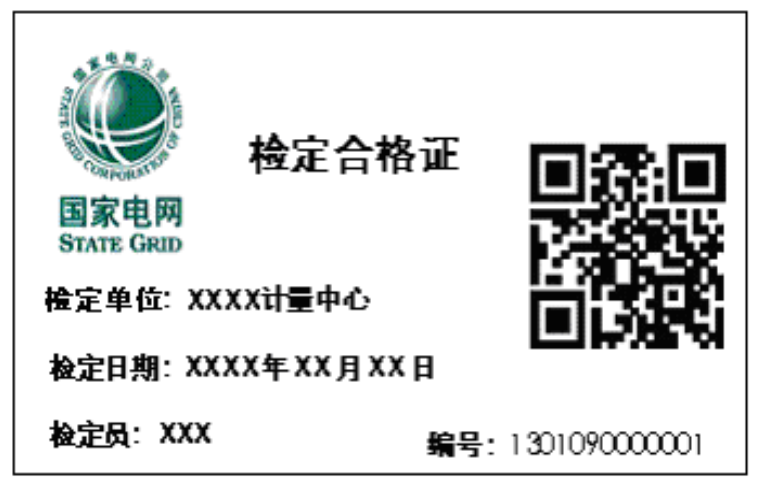

Fig1: Smart energy meter automatic calibration line verification seal label layout design

\section{B. The design of the calibration certificate}

Verification seal of smart energy meter left for the verification certificate information, including the smart energy meter calibration unit, date of calibration and verification personnel information. Seal bar code label is on the right side of the smart energy meter verification seal of $2 \mathrm{~d}$ barcode.

\section{Seal of preventing electricity-stolen function design}

Verification seal adopts bring back adhesive label materials, verification seal label on the upper left corner is to use laser technology to make the flag icon of State Grid, this icon is a can show company's corporate image, and it is made by laser technology can prevent from taking pictures imitation this verification seal.

Verification seal label the back plastic anti-fake design technology, the label after the tear leaves " Power theft is illegal" the obvious signs and marks, and can no longer be restored. Label paste is printed with " Power theft is illegal " of the two-dimensional glue, when the label after the tear from glued object, two-dimensional glue automatic separation, which borders the word d glue with labels and separation of glued objects become transparent. Another d residue on the surface of the object that is glued on, if we stick to object would have clear lines, so once the label was damaged it will be difficult to recover. If the object will be glued on the residual glue scrape and scrub clean.

\section{Invisible anti-counterfeiting design}

In verification to certain parts of the seal label printed with invisible anti-counterfeiting icon as the label of the anticounterfeiting mark, invisible bar code under the natural light is not visible, so on the label add invisible anti-counterfeiting icon can make general counterfeiters are difficult to copy, achieve good anti-counterfeit effect, also won't destroy the overall design and appearance of the calibration label effect. Icon invisible way in principle there are three: one is hiding cover type, the logo printing is completed, then a particular film or coating should be covered, normally can't see, 2 it is to use photochemical processing, by photochemical technology to deal with printing logo, after processing under a specific light source cannot recognition for icon; Three is to use an invisible ink, using invisible ink printing, adopt and ordinary process for printing. The seal label is invisible ink printing way. First smart energy meter calibration line labeling computer will automatically calculate the contact icon to print on the label, then add a fluorescent dye fluorescent ink through aerosol spray jet printer on a verification on the label, this kind of method to print out the contact icon, with the naked eye is invisible, only at a certain frequency band, under the irradiation of near infrared laser to see hidden ICONS and other security information.

\section{E. Automatic labeling process design}

Automatic labeling institutions with high precision, fast set of standard device, through the high accuracy intelligent electric eye and high reliable microcomputer chip program controller (PLC) control servo motor of label, with 60 set of parameters stored and counting function, not only of label position precision is high, and the shrinkage is more appropriate to be posted after the appearance of objects. The key to the design and the mechanical priciple of the labelling machine is PLC control. In the process of design, the technology of sensor, computer control and pneumatic is applied. The labelling speed is reached 500/min.

After completion of the verification work, the calibration qualified smart energy meter without manual sealing containers or paste the verification certificate, automatic labeling institutions from the database read sequentially sealing bar code information, read the current date code values 
and verification personnel information, and put them on the seal label printing, automatic labeling institutions will print again good seal label paste where indicated on the smart energy meter automatically, and then through the recognizer to check the result of the label. Recognizer scanning determination smart energy meter and, if the label is not successful, the smart energy meter through the back to make the delivery to automatic labeling institutions to labeling. Its efficiency in reserve satisfies the requirement of verification system on the basis of more than $20 \%$ of the redundancy. Labeling rate greater than $99.5 \%$, labeling integrity recognition accuracy is $100 \%$. Specific work flow is as follows:

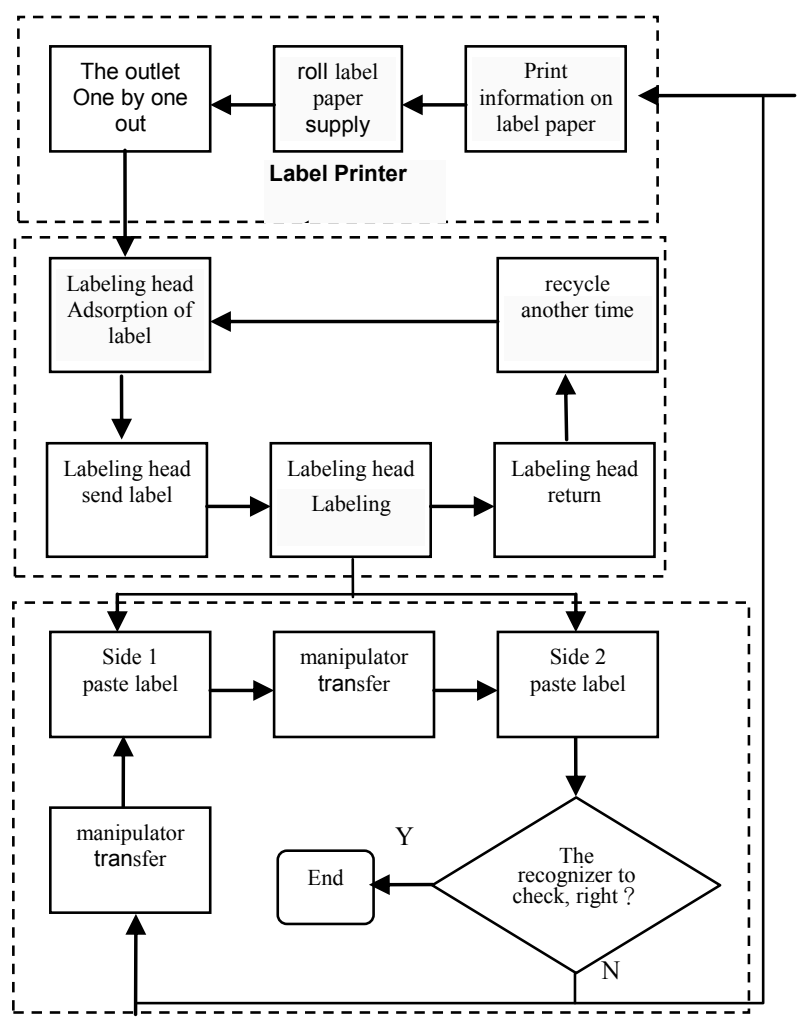

Fig2:Automatic labeling work flow chart

\section{The design of the bar code encryption}

Because of the characteristic of the two-dimensional bar code is the large capacity of information, information density of two-dimensional bar code PDF417 is twenty times more than one-dimensional Code39 bar code, able to ASCII, characters, images, etc all can the encoded after the digital information portable data files, can be effectively passed directly, need not be as one-dimensional bar code and with the help of a database support to find out more detailed information. As a result, the verification seal of preventing electricity-stolen seal bar code uses a $2 \mathrm{~d}$ barcode.

\section{A. The encryption algorithm}

TABE1: Seal the original bar code signal encryption process table

\begin{tabular}{|c|c|c|c|c|c|c|}
\hline Steps & Methods & $\begin{array}{l}\text { Check } \\
\text { code }\end{array}$ & $\mathrm{X} 1$ & $\mathrm{X} 2$ & ...... & $\mathrm{Xn}$ \\
\hline 1 & $\begin{array}{l}\text { The original value of } \\
\text { the bar code }\end{array}$ & C & 1 & 2 & $?$ & 3 \\
\hline 2 & $\begin{array}{l}\text { Take of digits } \\
\text { corresponding } \\
\text { weighted value } \\
\end{array}$ & & 3 & 1 & ....... & 2 \\
\hline 3 & $\begin{array}{l}\text { Would you number } \\
\text { corresponding } \\
\text { weighted value } \\
\text { multiplied in turn }\end{array}$ & & 3 & 2 & Y & 6 \\
\hline 4 & $\begin{array}{l}\text { Addition of product, } \\
\text { and several are } \\
\text { obtained }\end{array}$ & & \multicolumn{4}{|c|}{$11+\mathrm{Y}$} \\
\hline 5 & $\begin{array}{l}\text { With the sum divided } \\
\text { by the modulus of } 10, \\
\text { draw a remainder }\end{array}$ & & \multicolumn{4}{|c|}{ M } \\
\hline 6 & $\begin{array}{l}\text { Modulus decrease } 10 \\
\text { remainder, the } \\
\text { difference is the check } \\
\text { code }\end{array}$ & & \multicolumn{4}{|c|}{$K=10-M$} \\
\hline 7 & $\begin{array}{l}\text { Will check at the end } \\
\text { of the form id code } \\
\text { pattern }\end{array}$ & K & 1 & 2 & $\ldots \ldots$ & 3 \\
\hline
\end{tabular}

Smart energy meter automatic verification when automatic print and paste the verification seal assembly line, the first is to control the computer depending on the type of seal to print the original bar code, serial number, etc. Property, two-dimensional bar code structure and the generation of code value principles refer to bar code compiled rules "marketing business application system", with the verification seal to the original value of the bar code, can be to generate $2 \mathrm{~d}$ barcodes encryption to encrypt its value. Indicated upper table simply introduces an encryption method and the encryption process.

\section{B. Reverse power smart energy meter seal bar code read on} the site

Bar code tracing verification seal identification system using dedicated terminal PDA scanning, using SQLCE and C \# programming technology to develop the special decryption software. By PDA scanning bar code information will be read in, and then through declassification procedures show the verification seal the real content of bar code. If it is to use other scanners scanned can win the cipher of the bar code content and cannot know the real code value of the bar code.

\section{Verification seal design characteristics}

1) Seal card left upper corner of the label is with laser technology to make its icon, is shows the company's corporate image, and can prevent the pirated copy through photo technology.

2 ) Will smart energy meter calibration and verification certificate synthesis of seal, the automatic verification assembly line of the seal verification, verification certificate to print and paste to synthesize into a process, optimize the process and process, saved equipment investment. 
3 ) Seal certificate label the back glue anti-counterfeiting technology, tear leaves after " Power theft is illegal" clearly marked and the mark, there is no recovery. Can be found residual glue labels is clear to the eye.

4 ) Seal certificate label bar-code and smart energy meter asset number through verification on the assembly line automatically binding to marketing system, to provide basic data for electricity in the future inspection.

5) Used the invisible anti-counterfeiting design, only at a certain frequency band, under the irradiation of near infrared laser can see invisible ICONS and other security information.

6 ) Seal adopts the two-dimensional bar code encryption algorithm, developed the decryption software, only internal special bar code scanner using a special decryption software can read the correct code value. Other scanner read only is garbled. $2 \mathrm{~d}$ encryption source code bar code encoding rules refer to "marketing business bar code application system establishment rules".

\section{Conclusion}

Verification seal of smart energy meter is put into application in automatic calibration line of some electric power company metering center, and got the recognitions of the State Grid, the Provincial Bureau of Quality and Technical Supervision and other brothers units. In 2013, the measuring center will check the province's single-phase, three-phase smart energy meter, each smart energy meter will paste the 2 verification seal (one is measuring center, the other is for the Provincial Bureau of Quality and Technical Supervision), so the verification seal application prospect.

\section{References}

[1] GoldreiehOded.Foundationsoferyptography:basietools.Cambridge, U.K.Newyork: CambridgeUniversityPress, 2001

[2] TrappeWade.IntroduCtiontocryptography:witheodingtheory. UpperSaddleRiver, NJ:PrentieeHall, 2002.Pages:75-76

[3] JiShouWen MiaoLiXin, Li keqiang. Modern logistics equipment and technology practice [M]. Haitian press, in January 2004

[4] WeiYuanHua. Bar code technology and application [M]. China textile press books circulation department in Beijing, June 1, 2003

[5] Peng Huang. Bar code in the application of products production management research and development [M]. Nanjing aerospace university press, July 2005

[6] Yuan Weihua. The application of barcode technology in logistics management, the electronic industrial press, July 2005

[7] Wang. Anti-counterfeiting technology, Chemical industry press, August 2003 